Sādhanā Vol. 40, Part 4, June 2015, pp. 1091-1104. (C) Indian Academy of Sciences

\title{
Extraction of fetal electrocardiogram (ECG) by extended state Kalman filtering and adaptive neuro-fuzzy inference system (ANFIS) based on single channel abdominal recording
}

\author{
D PANIGRAHY* and P K SAHU
}

Department of Electrical Engineering, NIT Rourkela, Rourkela 769008, India e-mail:damo.3093@gmail.com; pksahu@nitrkl.ac.in

MS received 11 February 2014; revised 9 December 2014; accepted 23 January 2015

\begin{abstract}
Fetal electrocardiogram (ECG) gives information about the health status of fetus and so, an early diagnosis of any cardiac defect before delivery increases the effectiveness of appropriate treatment. In this paper, authors investigate the use of adaptive neuro-fuzzy inference system (ANFIS) with extended Kalman filter for fetal ECG extraction from one ECG signal recorded at the abdominal areas of the mother's skin. The abdominal ECG is considered to be composite as it contains both mother's and fetus' ECG signals. We use extended Kalman filter framework to estimate the maternal component from abdominal ECG. The maternal component in the abdominal ECG signal is a nonlinear transformed version of maternal ECG. ANFIS network has been used to identify this nonlinear relationship, and to align the estimated maternal ECG signal with the maternal component in the abdominal ECG signal. Thus, we extract the fetal ECG component by subtracting the aligned version of the estimated maternal ECG from the abdominal signal. Our results demonstrate the effectiveness of the proposed technique in extracting the fetal ECG component from abdominal signal at different noise levels. The proposed technique is also validated on the extraction of fetal ECG from both actual abdominal recordings and synthetic abdominal recording.
\end{abstract}

Keywords. Adaptive neuro-fuzzy inference systems (ANFIS); extended Kalman filter (EKF); extended Kalman smoother (EKS); fetal electrocardiogram.

\section{Introduction}

Fetal heart rate (FHR) monitoring is a routine work for obtaining significant information about the fetal condition during pregnancy and labor. During pregnancy, the motivation for monitoring the fetal is to recognize pathological conditions, typically asphyxia with sufficient warning to enable intervention by the clinician. So FHR carries significant importance of clinical perspective (Hasan et al 2007).

$\overline{* \text { For correspondence }}$ 
There are two most popular techniques used by which one can monitor FHR are ultrasound and fetal electrocardiogram (ECG). In ultrasound, a doctor moves a device that looks roughly like a hockey puck over a woman's abdomen or she wears a belt fitted with sensors to detect heartbeat quite early in pregnancy. However, it is not suitable for long period of FHR monitoring. It is sensitive to movement and also not suitable for beat-to-beat analysis as detection of the QRS complex. QRS complex represents the ventricular depolarization prior to their contraction of heart.

The fetal ECG reflects the electrical activity of fetus. It contains the characteristics of the fetus such as heart rate, signal waveform and dynamic behaviours which help in determining the fetal life, fetal development, fetal maturity and existence of fetal distress or congenital heart disease, etc. So by observing above characteristics appropriate treatment is usually performed (Deans \& Steer 1994). There are several technical problems associated with the non-invasive extraction of fetal ECG from ECG signals recorded at abdominal surface. These problems are mainly due to the low power of the fetal ECG signal which is contaminated by various sources of interference. These sources include the maternal ECG, the maternal electromyogram (EMG), $50 \mathrm{~Hz}$ power line interference, baseline wander and random electronic noise (Zeng et al 2008; Ferrara \& Widrow 1982). By using low noise electronic amplifiers with high common mode rejection ratio, the effect of the $50 \mathrm{~Hz}$ interference and electronic random noise can be eliminated. The EMG noise can also be reduced but not necessarily required to be eliminated with the use of classical low pass filtering techniques. Hence, one can conclude that the elimination of the maternal ECG component from the composite signal produces a reasonable estimate of the fetal ECG signal.

Several methods have been proposed for detecting and processing the fetal ECG signal from the abdominal ECG signal. These proposed techniques include discrete Fourier transform method (Tal \& Akselrod 1989), adaptive filter (Ferrara \& Widrow 1982), correlation techniques (Abboud et al 1992), singular value decomposition (Callaerts et al 1990; Kanjilal et al 1997), sequential processing method (modified template subtraction) (Arias-Ortega \& Gaitán-González 2010), non-parametric method (Niknazar et al 2012), wavelet transform (Mochimaru 2002; Khamene \& Negahdaripour 2000), neural network technique (Camps et al 2001; Camps-Valls et al 2004), polynomial network (Assaleh \& Al Nashash 2005), adaptive neuro-fuzzy inference system (ANFIS) (Assaleh 2007), and Blind Source Separation (BSS) (De Lathauwer et al 2000; Zarzoso et al 1997), BSS via Independent Component Analysis (ICA) was considered to be the most successful method for fetal ECG extraction (Zarzoso \& Nandi 2001). However in order for ICA to work properly, it requires multiple leads for collecting several ECG signals in contrast to single abdominal lead as proposed in this work. Certainly not enough satisfactory fetal ECG extraction via ICA have been reported, because ICA assumes the linear relationship between maternal ECG and maternal component in abdominal ECG. But the actual relationship between maternal ECG and maternal component in abdominal ECG is nonlinear. Other techniques (Ferrara \& Widrow 1982; Camps et al 2001) also do not give satisfactory results when single abdominal ECG signal is used. In this work, we have proposed a new fetal ECG extraction technique based on the extended Kalman filter (Sameni Reza 2008; Niknazar Mohammad et al 2013), which shows encouraging results on extracting the fetal ECG from single channel abdominal ECG recording.

In this paper, we aim to apply ANFIS with an extended Kalman filter framework for estimating fetal ECG component from one abdominal ECG recording. We have used ANFIS to align with estimated maternal ECG from abdominal ECG. This nonlinear alignment between two signals allows us for cancelling the maternal component from the abdominal ECG signal and hence offers an estimate of the fetal ECG signal. Results shown in this paper have been validated with synthetic as well as real ECG data. We have specifically shown some analysis and 


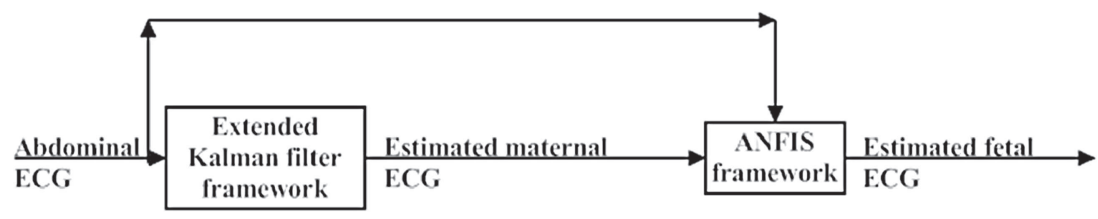

Figure 1. Block diagram of proposed method.

comparative results on the proposed method (EKS+ANFIS, EKF+ANFIS) with five other techniques like Extended Kalman Filter (EKF) (Sameni 2008), Extended Kalman Smoother (EKS) (Sameni 2008), non-parametric method (Niknazar et al 2012), sequential processing method (modified template subtraction) (Arias-Ortega \& Gaitán-González 2010) and Singular Value Decomposition (SVD) (Kanjilal et al 1997) to claim the better accuracy of our work.

Organisation of the paper is as follows: In section 2, methodology of our proposed technique including Bayesian filtering theory (extended Kalman filter), ANFIS and dynamic ECG model are described. In section 3, results of proposed method applied on different data and discussions thereof are presented. Finally, our conclusion is stated in section 4.

\section{Methodology}

We use extended Kalman filter framework to estimate the maternal component from abdominal ECG. In the next step, an ANFIS network is used to identify this nonlinear relationship and to align the estimated maternal ECG signal with the maternal component in the abdominal ECG signal. Thus we extract the fetal ECG component by subtracting the aligned version of the estimated maternal ECG from the abdominal ECG. In subsection 2.1, the extended Kalman filter framework to estimate maternal ECG from abdominal ECG has been presented. In subsection 2.2, the ANFIS framework to estimate fetal ECG from estimated maternal ECG is explained. Figure 1 shows the block schematic representation of proposed technique.

\subsection{Extended Kalman filter framework}

The goal of the extended Kalman filter is to estimate the state of a discrete time controlled process. Consider a state vector $\mathrm{X}_{\mathrm{K}+1}$ governed by a nonlinear stochastic difference equation with measurement vector $\mathrm{Y}_{\mathrm{K}+1}$ at time instant $\mathrm{K}+1$ (Welch \& Bishop 2006):

$$
\begin{gathered}
\mathrm{X}_{\mathrm{K}+1}=\mathrm{f}\left(\mathrm{X}_{\mathrm{K}}\right)+\mathrm{W}_{\mathrm{K}} \\
\mathrm{Y}_{\mathrm{K}+1}=\mathrm{h}\left(\mathrm{X}_{\mathrm{K}+1}\right)+\mathrm{V}_{\mathrm{K}+1},
\end{gathered}
$$

where $\mathrm{W}_{\mathrm{K}}$ and $\mathrm{Vă}_{\mathrm{K}}$ represent process and measurement noises, $\mathrm{f}($.$) defines system dynamic and$ $\mathrm{h}($.) defines measurement function. In this study, a synthetic dynamic ECG model (McSharry et al 2003) is used to estimate maternal ECG from abdominal ECG. One ECG signal can be expressed as the sum of five Gaussian functions defined by their peak amplitudes, width and center denoted as $\alpha_{\mathrm{i}}, \mathrm{b}_{\mathrm{i}}, \phi_{\mathrm{i}}$ respectively

$$
\mathrm{Z}(\theta)=-\sum_{\mathrm{i} \in \mathrm{W}} \alpha_{\mathrm{i}} \Delta \theta_{\mathrm{i}} \exp \left(\frac{-\left(\Delta \theta_{\mathrm{i}}\right)^{2}}{2 \mathrm{~b}_{\mathrm{i}}{ }^{2}}\right),
$$

where $\Delta \theta_{\mathrm{i}}=\left(\theta-\phi_{\mathrm{i}}\right) \bmod (2 \pi)$ 
Each Gaussian function models one of the five waves $\mathrm{w}=\{P, Q, R, S, T\}$ of heart beat.

The state vector $X_{K}$ in Eq. (1) is defined as $X_{K}=\left[\begin{array}{ll}\theta_{K} & Z_{K}\end{array}\right]^{T}$ where $\theta$ is the phase and $Z$ is the amplitude of the ECG. Assuming small sampling period, the state process $\mathrm{f}($.$) is$

$$
\theta_{\mathrm{K}+1}=\left(\theta_{\mathrm{K}}+w \delta\right) \bmod (2 \pi) \text {. }
$$

Here $\delta=$ small sampling period.

$$
\mathrm{Z}_{\mathrm{K}+1}=-\sum_{\mathrm{i} \in w} \frac{\alpha_{\mathrm{i}} \mathrm{w}_{\mathrm{f}} \delta \Delta \theta_{\mathrm{i}, \mathrm{K}}}{\mathrm{b}_{\mathrm{i}}^{2}} \exp \left(\frac{-\Delta \theta_{\mathrm{i}, \mathrm{K}}^{2}}{2 \mathrm{~b}_{\mathrm{i}}^{2}}\right)+\mathrm{Z}_{\mathrm{K}},
$$

where $\mathrm{w}_{\mathrm{f}}$ is the phase increment and $\Delta \theta_{\mathrm{i}, \mathrm{K}}=\left(\theta_{\mathrm{K}}-\emptyset_{\mathrm{i}}\right) \bmod (2 \pi)$.

Equation (1) represents the state equation and description of state equation is given above. The next step of the extended Kalman filter is an observation equation as represented in Eq. (2).

Observation data $\mathrm{Y}_{\mathrm{K}+1}=\left[\begin{array}{ll}\varphi_{\mathrm{K}+1} & \mathrm{~S}_{\mathrm{K}+1}\end{array}\right]^{\mathrm{T}}$ where $\varphi_{\mathrm{K}+1}=$ phase of ECG which is between $\left[\begin{array}{ll}-\pi & \pi\end{array}\right], \mathrm{S}_{\mathrm{K}+1}$ is the amplitude of abdominal ECG at instant or sample number $\mathrm{K}+1$. We can determine $\varphi_{\mathrm{K}+1}$ by first estimating R peaks of ECG signals actually these R peaks are maternal ECG R peaks. Phase $\varphi_{\mathrm{K}+1}$ is assigned as zero for each R peak. Observed phase other than R peak can be estimated by a linear time warping of the $R-R$ intervals between $[-\pi \pi]$. The assignment of phase and abdominal ECG signal is represented in figure 2. The measurement function $\mathrm{h}($.$) is defined as \mathrm{X}_{\mathrm{K}+1}$.

The next step of the extended Kalman filter is the initialization. $\widehat{\mathrm{X}_{0}}$ and $\mathrm{P}_{0}$ are initialized as: $-\widehat{\mathrm{X}_{0}}=[-\pi, 0]^{\mathrm{T}}$.

$$
\mathrm{P}_{0}=\left[\begin{array}{cc}
(2 \pi)^{2} & 0 \\
0 & 10 \max (|\mathrm{S}|)^{2}
\end{array}\right] .
$$

The final step of the extended Kaman filter is the computation. The description of computation is as follows

For $\mathrm{K}=0,1,2,3, \ldots \mathrm{N}$

$$
\overline{\mathrm{X}_{\mathrm{K}+1}}=\mathrm{f}\left(\widehat{\mathrm{X}_{\mathrm{K}}}\right)
$$

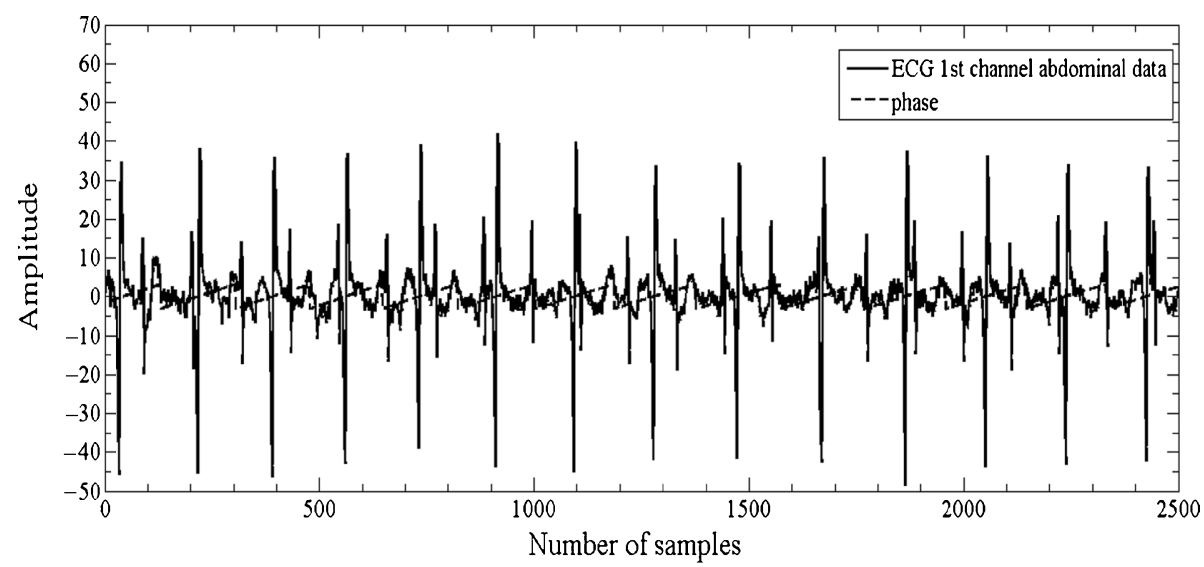

Figure 2. Phase assignment approach of abdominal ECG of $1^{\text {st }}$ channel DaISY database (Moor et al 1997). 


$$
\begin{aligned}
& \overline{\mathrm{P}_{\mathrm{K}+1}}=\nabla \mathrm{f}_{\mathrm{X}} \mathrm{P}_{\mathrm{K}} \nabla \mathrm{f}_{\mathrm{X}}^{\mathrm{T}}+\mathrm{Q} \\
& \widehat{\mathrm{X}_{\mathrm{K}+1}}=\overline{\mathrm{X}_{\mathrm{K}+1}}+\mathrm{Kg}\left(\mathrm{Z}_{\mathrm{K}+1}-\mathrm{h}\left(\overline{\mathrm{X}_{\mathrm{K}+1}}\right)\right) \\
& \text { As } h(.)=X_{K+1} \text { So } \widehat{X_{K+1}}=\overline{X_{K+1}}+K g\left(Z_{K+1}-\overline{X_{K}+1}\right) \\
& \mathrm{S}=\nabla \mathrm{h} \overline{\mathrm{P}_{\mathrm{K}+1}} \nabla \mathrm{h}^{\mathrm{T}}+\mathrm{R} \\
& \mathrm{Kg}=\overline{\mathrm{P}_{\mathrm{K}+1}} \nabla \mathrm{h}^{\mathrm{T}} \mathrm{S}^{-1} \\
& \mathrm{P}_{\mathrm{K}+1}=\overline{\mathrm{P}_{\mathrm{K}+1}}-\left((\mathrm{Kg}) \mathrm{S}\left(\mathrm{Kg}^{\mathrm{T}}\right)\right) \\
& \text { Here } \nabla f_{\mathrm{x}}=\left[\begin{array}{cc}
1 & 0 \\
\sum_{i \in w} \frac{\alpha_{\mathrm{i}} \mathrm{w}_{\mathrm{f}} \delta}{b_{\mathrm{i}}^{2}}\left[1-\frac{\Delta \theta_{\mathrm{i}}^{2}}{b_{\mathrm{i}}^{2}}\right]-\exp \left(\frac{-\Delta \theta_{\mathrm{i}}^{2}}{2 \mathrm{~b}_{\mathrm{i}}^{2}}\right) & 1
\end{array}\right] \\
& \nabla \mathrm{h}=\left[\begin{array}{ll}
1 & 0 \\
0 & 1
\end{array}\right]
\end{aligned}
$$

$\mathrm{Q}$ and $\mathrm{R}$ are the covariance of process noise and measurement noise respectively which is calculated from mean and standard deviation of the abdominal ECG (Sameni et al 2007), $\mathrm{f}($.) is described in Eqs. (4) and (5) and $h()=.X_{K+1}$. N= length of abdominal ECG data. $\overline{X_{K+1}}$ is the estimated maternal ECG consisting of 2 rows. In the estimated maternal ECG first row corresponds to phase of the estimated component and second row corresponds to amplitude. The above process is known as EKF. EKS uses future information to provide better estimates of the current state. Due to non-causal nature, the EKS is expected to have better performance than EKF. EKS algorithm basically consists of forward EKF stage followed by a backward recursive smoothing stage.

In extended Kalman filtering technique, sometimes operator interaction is required to set the initialization parameter depending upon the abdominal ECG signal. To avoid this problem, we use ANFIS on the output of the extended Kalman filter as maternal ECG component to linearly and nonlinearly align in abdominal signal and minimize error without operator interaction. Hence, ANFIS framework has been applied to estimate fetal ECG by using estimated maternal component and abdominal ECG.

\subsection{ANFIS framework}

There are several advantages in using ANFIS for pattern learning and detection as compared to linear systems and neural networks. These features are centered on the fact that ANFIS combines the capabilities of both neural networks and fuzzy systems in learning nonlinearities. Fuzzy techniques incorporate information sources into a fuzzy rule base that represents the knowledge of the network structure so as to accomplish easy structure learning techniques. Moreover, ANFIS architecture requirements and initializations are fewer and simpler compared to neural networks which require extensive trials and errors for optimization of their architecture and initializations (Kakar et al 2005).

Figure 3 shows the nonlinear nature of the maternal ECG in abdominal ECG. So, ANFIS is applied to estimate fetal ECG from abdominal ECG by using estimated maternal ECG.

To present the ANFIS architecture, let us consider two-fuzzy rule based system on a first order Sugeno model.

Rule 1: if $\left(X\right.$ is $\left.A_{1}\right)$ and $\left(Y\right.$ is $\left.B_{1}\right)$ then $f_{1}=p_{1} X+q_{1} Y+r_{1}$

Rule 2: if $\left(X\right.$ is $\left.A_{2}\right)$ and $\left(Y\right.$ is $\left.B_{2}\right)$ then $f_{2}=p_{2} X+q_{2} Y+r_{2}$. 


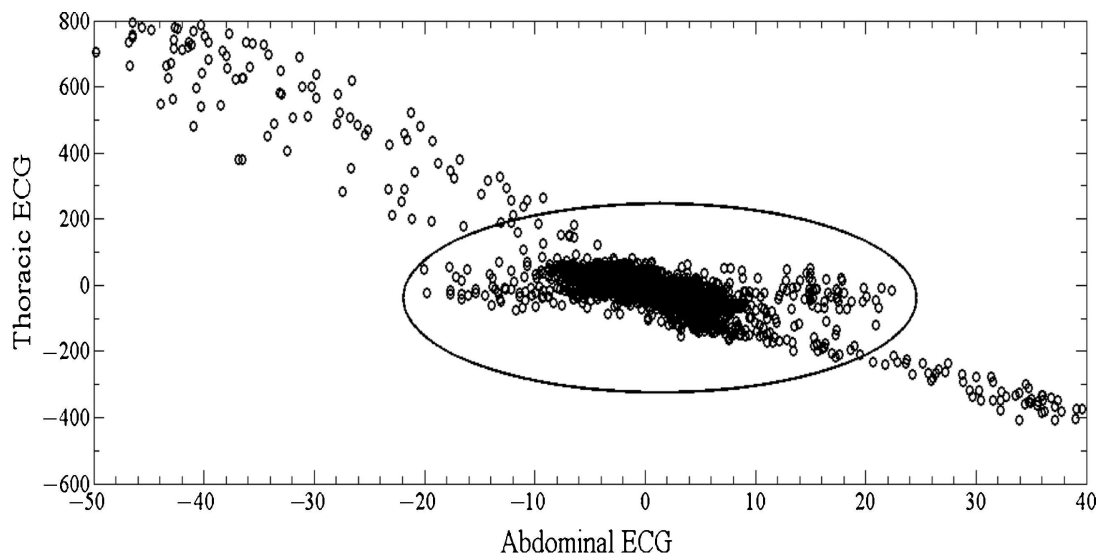

Figure 3. Relation between maternal ECG and abdominal ECG of DaISY data base (Moor et al 1997).

One possible ANFIS architecture to implement these two rules is shown in figure 4. Circles indicate fixed nodes and squares represent adaptive nodes. In the following presentation $\mathrm{O}_{\mathrm{Li}}$ denotes the output of node $\mathrm{i}$ in a layer $\mathrm{L}$.

$$
\mathrm{O}_{1, \mathrm{i}}=\left\{\begin{array}{l}
\mu A_{\mathrm{i}}(\mathrm{X})-\mathrm{i}=1,2 \\
\mu B_{\mathrm{i}-2}(\mathrm{Y})-\mathrm{i}=3,4
\end{array}\right\} .
$$

Here we use bell shape based membership function.

$$
\mu A_{\mathrm{i}}(\mathrm{X})=\frac{1}{1+\left[\left(\frac{\mathrm{X}-\mathrm{c}_{\mathrm{i}}}{\mathrm{a}_{\mathrm{i}}}\right)^{2}\right]^{\mathrm{b}_{\mathrm{i}}}} \quad \mathrm{i}=1,2
$$

$\mathrm{a}_{\mathrm{i}}, \mathrm{b}_{\mathrm{i}}, \mathrm{c}_{\mathrm{i}}$ are called the premises parameters.

$$
\mathrm{O}_{2, \mathrm{i}}=\mathrm{w}_{\mathrm{i}}=\mu A_{\mathrm{i}}(\mathrm{X}) \mu B_{\mathrm{i}}(\mathrm{Y}) \quad \mathrm{i}=1,2
$$

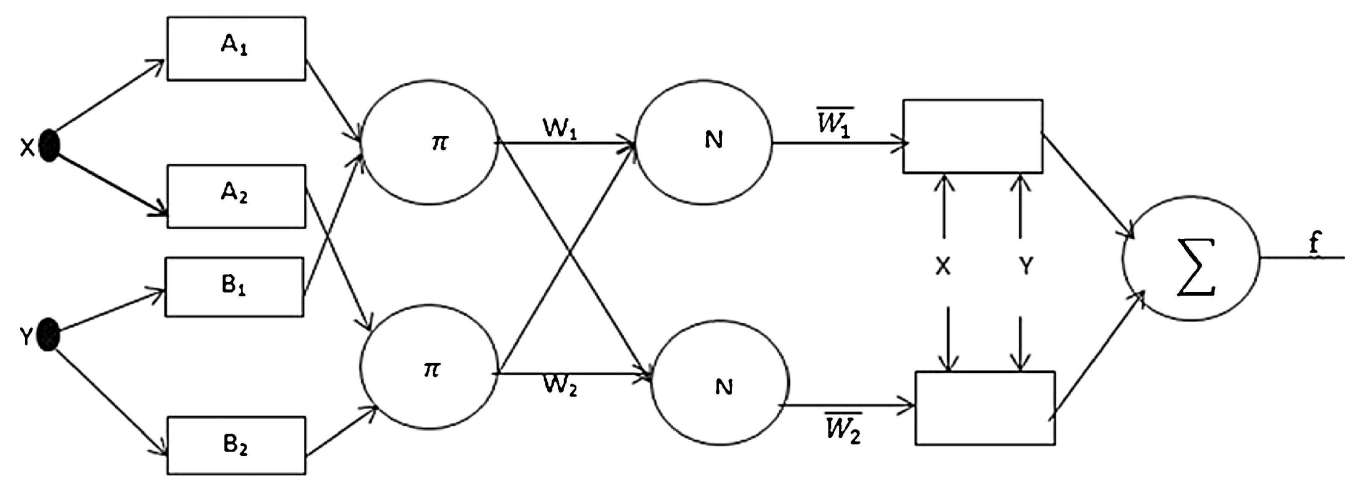

Figure 4. ANFIS architecture. 


$$
\begin{gathered}
\mathrm{O}_{3, \mathrm{i}}=\overline{\mathrm{w}_{\mathrm{i}}}=\frac{\mathrm{w}_{\mathrm{i}}}{\mathrm{w}_{1}+\mathrm{w}_{2}} \quad \mathrm{i}=1,2 \\
\mathrm{O}_{4, i}=\overline{\mathrm{w}_{\mathrm{i}}} \mathrm{f}_{\mathrm{i}}=\overline{\mathrm{w}_{\mathrm{i}}}\left(\mathrm{p}_{\mathrm{i}} \mathrm{X}+\mathrm{q}_{\mathrm{i}} \mathrm{Y}+\mathrm{r}_{\mathrm{i}}\right) \quad \mathrm{i}=1,2
\end{gathered}
$$

$\mathrm{p}_{\mathrm{i}}, \mathrm{q}_{\mathrm{i}}, \mathrm{r}_{\mathrm{i}}$ are called as consequent parameters.

$$
\mathrm{O}_{5,1}=\mathrm{f}=\sum_{\mathrm{i}} \overline{\mathrm{w}_{\mathrm{i}}} \mathrm{f}_{\mathrm{i}} \quad \mathrm{i}=1,2 .
$$

Here premises parameters are updated by backward sweep as steepest descent algorithm; consequent parameters are updated by forward sweep as a Least Mean Square algorithm (LMS). Here $\mathrm{X}=$ estimated component of the maternal ECG in abdominal ECG and $\mathrm{Y}=$ one step delay of $\mathrm{X}$.

$$
\text { Estimated fetal ECG = abdominal ECG }-\mathrm{f},
$$

where $\mathrm{f}=$ output of ANFIS architecture.

We have used 2, 4, 6, 8, 10, 12 membership functions with different iterations. In ANFIS, only $\mathrm{A}_{1}, \mathrm{~A}_{2}$ (2 numbers of $\left.\mathrm{A}\right)$ and $\mathrm{B}_{1}, \mathrm{~B}_{2}$ (2 numbers of $\left.\mathrm{B}\right)$ are used as 2 membership functions. Similarly 4 numbers of $A\left(A_{1}, A_{2}, A_{3}, A_{4}\right)$ and 4 numbers of $B\left(B_{1}, B_{2}, B_{3}, B_{4}\right), 6$ numbers of $A$ and 6 numbers of $\mathrm{B}, 8$ numbers of $\mathrm{A}$ and 8 numbers of $\mathrm{B}, 10$ numbers of $\mathrm{A}$ and 10 numbers of $\mathrm{B}, 12$ numbers of $\mathrm{A}$ and 12 numbers of $\mathrm{B}$ are used to generate membership functions in ANFIS known as 4, 6, 8, 10, 12 membership function respectively. ANFIS having 8 membership functions with 100 iterations produced a best estimate of fetal ECG signal.

\section{Results and discussion}

Both synthetic and actual data have been used to study the performance of the proposed method. In Subsection 3.1, the output Signal to Noise Ratio (SNR) of different techniques like EKF, EKS with proposed method has been compared on synthetic ECG data. In subsection 3.2 effectiveness of the method on actual data has also been examined.

\subsection{Fetal ECG extraction and performance analysis on synthetic data}

Single channel synthetic abdominal ECG used in this study is based on single channel ECG dynamic model presented in (McSharry et al 2003). The fetal ECG signal s(n) is synthesized by using heart rate $140 \mathrm{bpm}$ and sampling frequency $250 \mathrm{~Hz}$. Similarly maternal ECG signal $x(\mathrm{n})$ is synthesized by using heart rate $70 \mathrm{bpm}$ and sampling frequency $250 \mathrm{~Hz}$. A model for generating the abdominal signal $w(\mathrm{n})$ is proposed as shown in figure 5.

Figure 5 shows the proposed method to generate synthetic fetal ECG. Here fmSNR is fetal to maternal signal noise ratio.

The maternal ECG signal $\mathrm{x}(\mathrm{n})$ when passes from heart to abdomen it suffers delay and nonlinear effect. Here $\widehat{\mathrm{x}(\mathrm{n})}$ is the maternal ECG component in abdominal ECG.

The reason for nonlinear nature of the maternal ECG in abdominal ECG signal is due to the fact that the signal is measured far away from its source (the mother's heart), so it encounters some nonlinear transformation as it travels to the abdominal area.

We have also got the nonlinear nature of the maternal ECG in abdominal data by using real database (DaISY database). Figure 3 shows a 2500 samples record of an abdominal ECG signal plotted against the corresponding maternal ECG or thoracic ECG both taken from DaISY data based on sample rate of 250 samples/s. The data points in the ellipse in the region around 


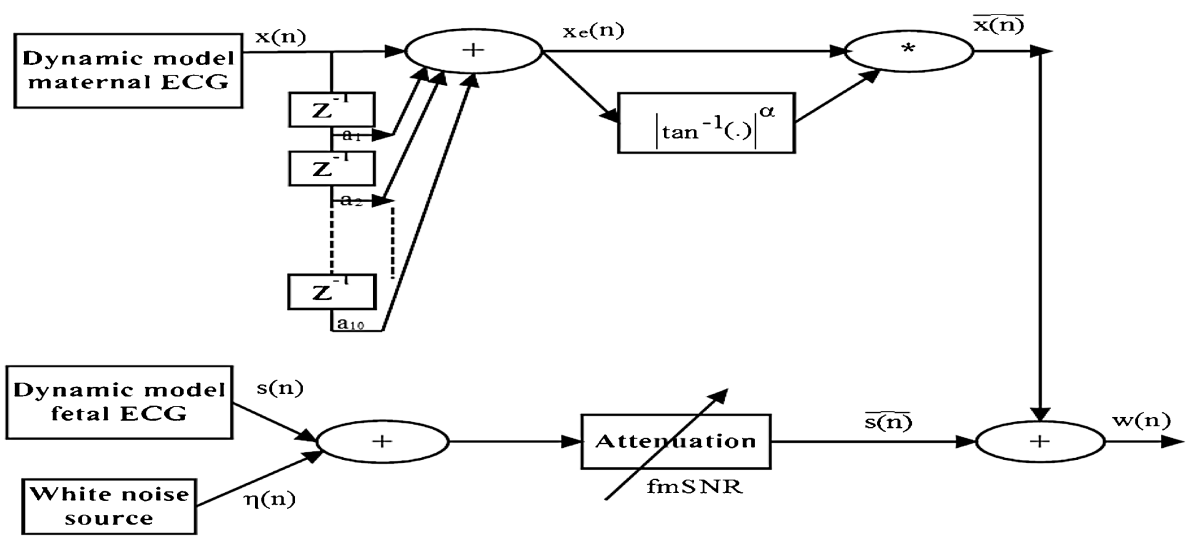

Figure 5. Block diagram for simulating abdominal ECG signal.

abdominal ECG signal between -20 to 20 and the maternal ECG signal is 0 corresponding to the QRS wave of the fetal ECG signal which exists in composite abdominal ECG signal. This shows the nonlinear nature of mapping between maternal ECG and maternal component in abdominal ECG by irregularity in the deviation of data points in the ellipse region.

So $\widehat{x(n)}$ is modelled as

$$
\widehat{\mathrm{x}(\mathrm{n})}=\left.\mathrm{x}_{\mathrm{e}}(n) \cdot \tan ^{-1}\left(\mathrm{x}_{\mathrm{e}}(\mathrm{n})\right)\right|^{\alpha},
$$

where

$$
\mathrm{x}_{\mathrm{e}}(n)=x(n)+\mathrm{a}_{1} x(n-1)+\mathrm{a}_{2} x(n-2)+\cdots+\mathrm{a}_{10} x(n-10) .
$$

We have chosen the parameter $\mathrm{a}_{\mathrm{i}}$ for $i=1,2, \ldots, 10$ by using hit and trial method to fit the abdominal ECG signal with DaISY database.

Here $\tan ^{-1}$ (.) is used to simulate the nonlinearities associated with the composite signal.

$$
\begin{gathered}
\text { So abdominal ECG }=w(n)=\widehat{x(n)}+\widehat{s(n)}, \\
\text { where } \widehat{s(n)}=s(n)+\eta(n) .
\end{gathered}
$$

We have chosen $\mathrm{a}_{1}=0.2, \mathrm{a}_{2}=0.1, \mathrm{a}_{3}=-0.5, \mathrm{a}_{4}=0.3, \mathrm{a}_{5}=0.2, \mathrm{a}_{6}=0.1, \mathrm{a}_{7}=0.4, \mathrm{a}_{8}=0.1, \mathrm{a}_{9}=0.2$, $\mathrm{a}_{10}=0.6$ and fetal to maternal signal noise ratio $=\mathrm{fmSNR}=-10 \mathrm{~dB}$

where fmSNR is calculated as

$$
\mathrm{fmSNR}=10 \log _{10}\left(\frac{\sum \widehat{\mathrm{s}(\mathrm{n})}^{2}}{\sum \widehat{\mathrm{x}(\mathrm{n})}^{2}}\right),
$$

3.1a Output waveform analysis: Figure 6 presents the generated synthetic abdominal ECG signal, estimated fetal ECG signal by using EKS with ANFIS on synthetic abdominal ECG signal and synthetic fetal ECG signal respectively. It is found that estimated fetal ECG signal by using EKS with ANFIS is $90 \%$ accurate as the synthetic fetal ECG signal.

3.1b SNR analysis: In order to validate the performance of proposed method, we have varied the noise power and computed the SNR.

$$
\mathrm{SNR}=\sum \frac{\mathrm{af^{2 }}}{(\mathrm{ef}-\mathrm{af})^{2}},
$$

where ef= estimated fetal $\mathrm{ECG}, \mathrm{af}=$ actual fetal $\mathrm{ECG}=\mathrm{s}(\mathrm{n})$. 
Synthetic abdominal ECG

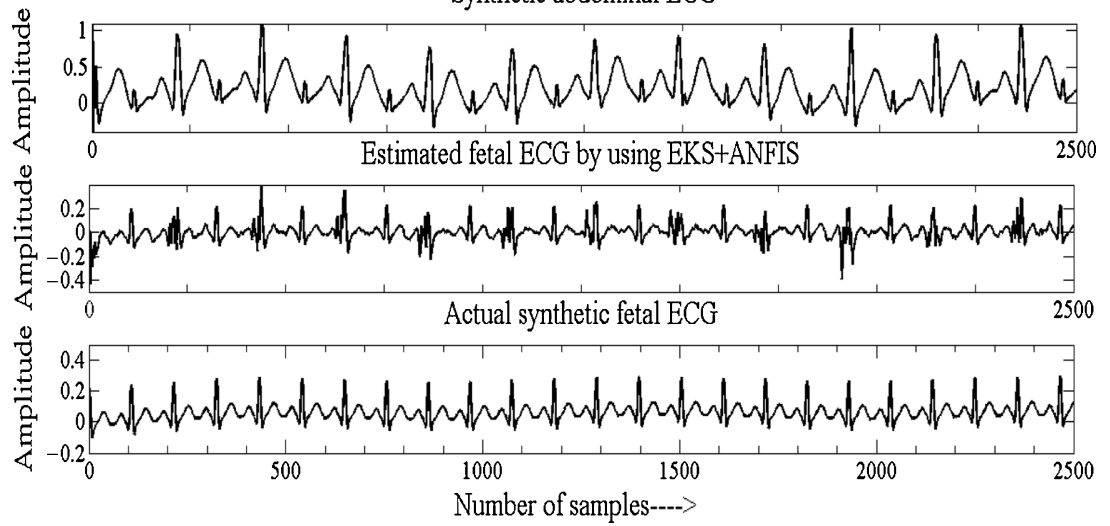

Figure 6. Comparison of estimated fetal ECG by EKS+ANFIS on synthetic ECG and actual fetal ECG.

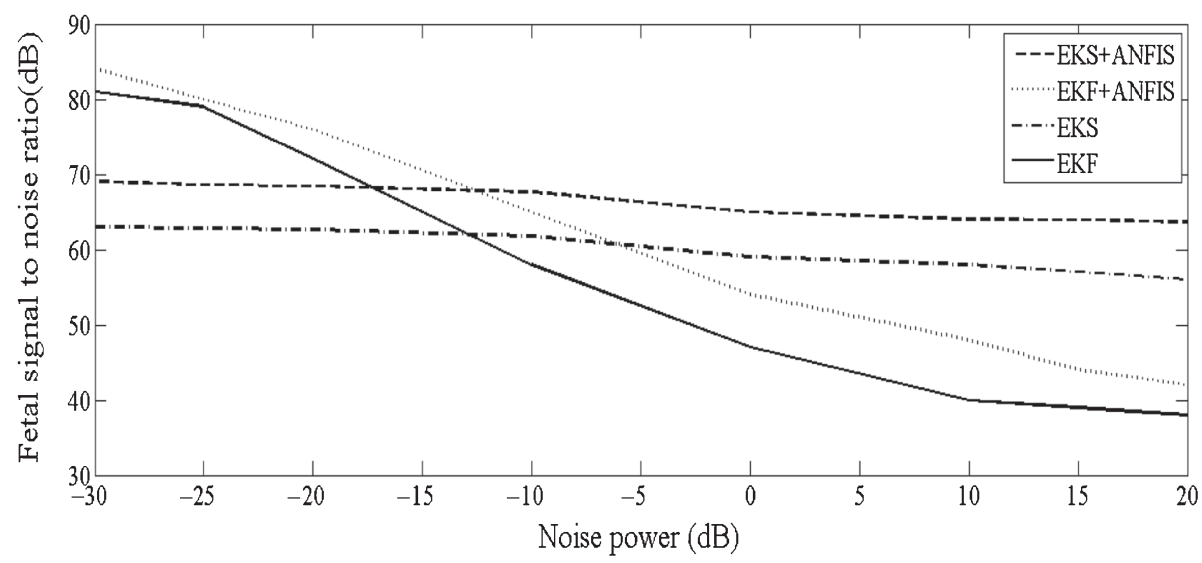

Figure 7. Comparison of estimated fetal ECG SNR of EKS+ANFIS, EKF+ANFIS, EKF+ANFIS, EKS, EKF by varying the noise level of synthetic ECG data.

Figure 7 shows the comparison of estimated fetal component by EKS+ANFIS, EKF+ANFIS, EKS, EKF on synthetic abdominal ECG data.

Figure 7 shows that even at noisy condition SNR of EKS+ANFIS give good fetal SNR which are not the case for other three methods.

\subsection{Fetal ECG extraction on actual data}

In this section, the results of application of the proposed technique on actual data are presented. Here we have considered two databases namely DaISY database and non-invasive fetal ECG database as a real data. In subsection 3.2a, we have shown some figures of estimated fetal ECG using EKS+ANFIS, EKF+ANFIS, EKS and EKF from DaISY database. In subsection 3.2b, we have calculated sensitivity (Se), positive predictive value (PPV) and accuracy of FHR detection of the proposed method and have compared the results with other techniques using non-invasive fetal ECG database. 
We have considered $60 \mathrm{~s}$ of abdominal data of non-invasive fetal ECG database to compute Se, PPV and accuracy. We have detected the R peak of the extracted fetal ECG from non-invasive fetal ECG using the technique as explained in the (Manikandan \& Soman 2012).

To evaluate the performance, we require three parameters namely true positive (TP), false negative (FN) and false positive (FP) from detected R peaks. Here TP is the number of correctly detected $\mathrm{R}$ peaks, FN is the number of missed $\mathrm{R}$ peaks. FP is the number of noise spikes detected as R peaks. Se, PPV and accuracy can be computed by using TP, FN, and FP.

$$
\begin{aligned}
\mathrm{Se} & =\frac{\mathrm{TP}}{\mathrm{TP}+\mathrm{FN}} \times 100 \% \\
\mathrm{PPV} & =\frac{\mathrm{TP}}{\mathrm{TP}+\mathrm{FP}} \times 100 \% . \\
\text { Accuracy } & =\frac{\mathrm{TP}}{\mathrm{TP}+\mathrm{FP}+\mathrm{FN}} \times 100 \% .
\end{aligned}
$$

We have manually selected 18 records $(18 \times 3=54$ channels $)$, where fetal QRS complex appeared to be the most visible after pre-processing step (removal of baseline wander and power line interference (Nallathambi \& Member 2014)) for computation of Se, PPV and accuracy using non-invasive fetal ECG database.

3.2a DaISY datadae: The ECG signals in DaISY dataset were recorded from eight different skin electrodes located on different points of a pregnant woman's body with $250 \mathrm{~Hz}$ sampling frequency. Five of these simultaneous signals were obtained from the mother's abdominal region while other three were obtained from the mother's thoracic region and each channel data consist of 2500 samples (Moor et al 1997).

Figures 8 and 9 present estimated fetal ECG by using EKS+ANFIS, EKF+ANFIS, EKS, EKF on the first and second channel DaISy data respectively. It is found that between 100 samples

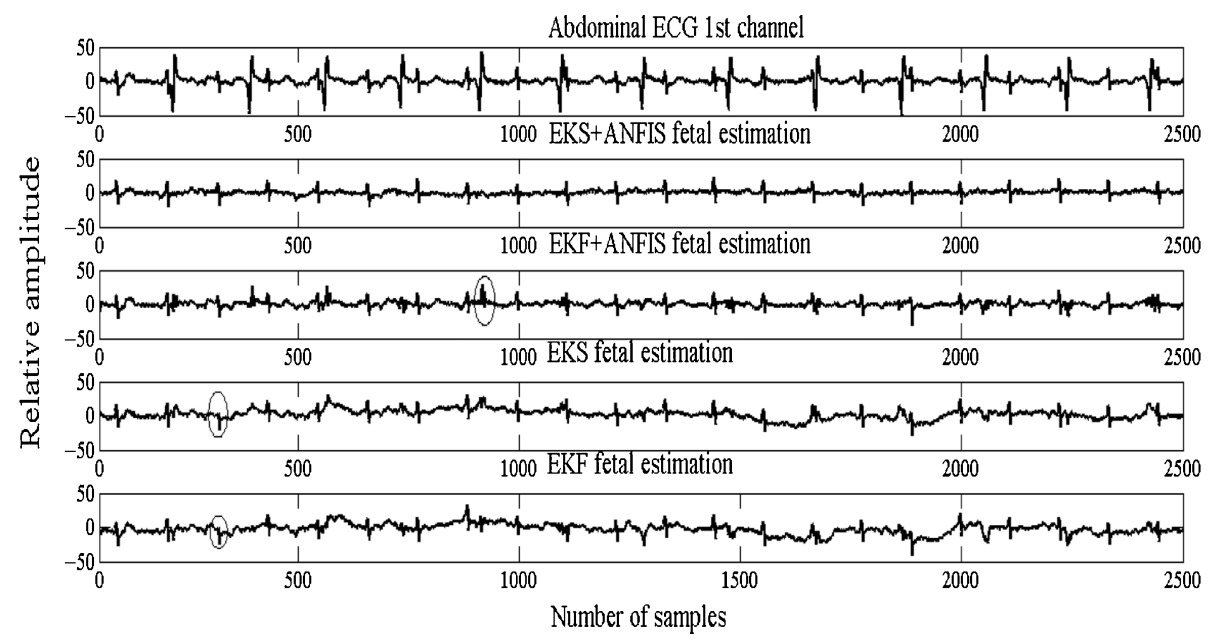

Figure 8. Comparison of estimated fetal ECG by EKS+ANFIS, EKF+ANFIS, EKS, EKF on first channel DaISy data. 


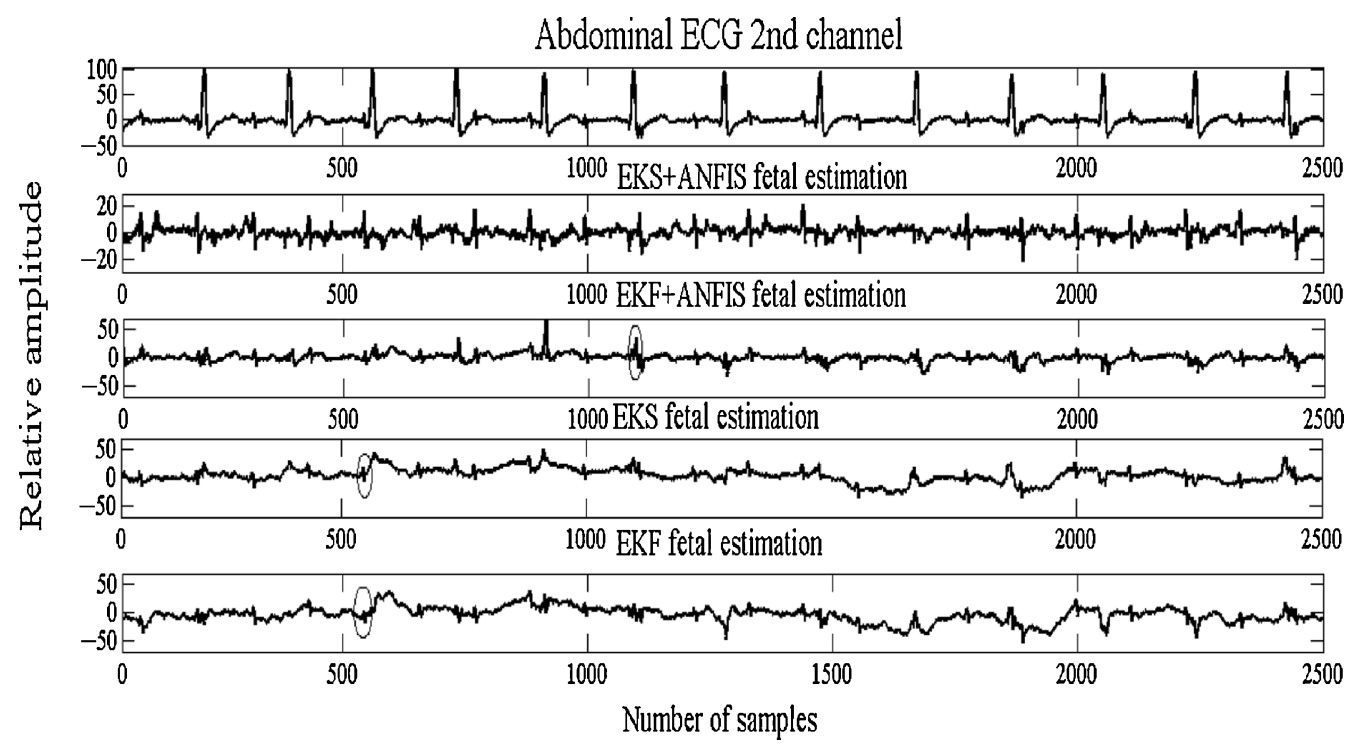

Figure 9. Comparison of estimated fetal ECG by EKS+ANFIS, EKF+ANFIS, EKS, EKF on second channel DaISy data.

and 500 samples of the estimated fetal ECG by using EKS and EKF on the first channel of DaISY database does not show the R peak of fetal. It is also found that between 800 samples and 1000 samples of the estimated fetal ECG by using EKF+ANFIS on the first channel of DaISY database does not cancel the maternal component. On the other hand EKS+ANFIS resulting in a better estimate of fetal ECG.

Similarly it is found that the estimated fetal ECG by using EKF and EKS on the second channel DaISY database does not show an R peak of fetal ECG between 500 samples and 700 samples. It is also found that the estimated fetal ECG by using EKF+ANFIS on the second channel DaISY database does not cancel maternal component between 800 samples and 1000 samples.

Finally figures 10 and 11 show the result of fetal ECG extraction using EKS+ANFIS applied on the third and fifth channels of the DaISY database. From the above analysis it is found that
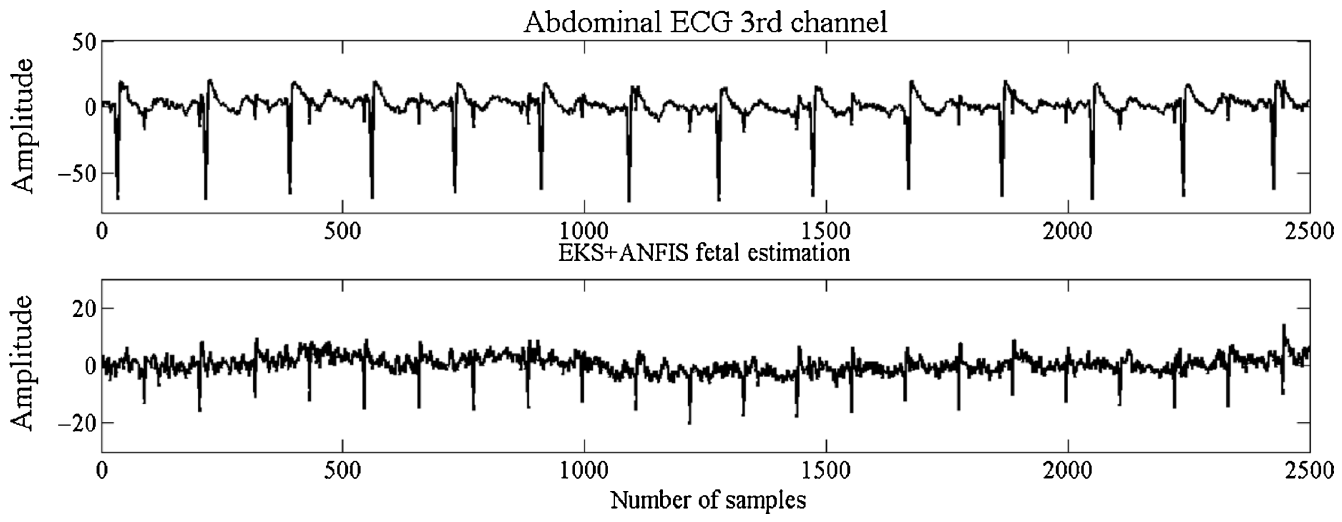

Figure 10. Result of fetal ECG extraction by using EKS+ANFIS on the third channel of DaISY database. 


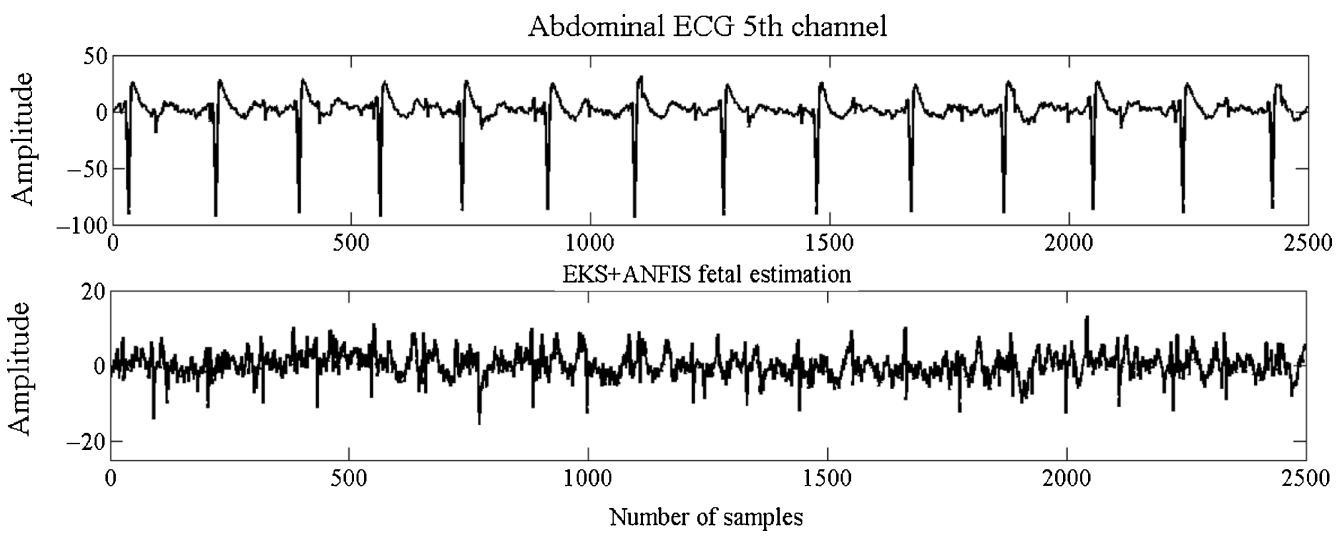

Figure 11. Result of fetal ECG extraction by using EKS+ANFIS on $5^{\text {th }}$ channel of DaISY database.

estimation of fetal ECG is encouraging even with ill-conditioned data which have been shown in figures 10 and 11.

3.2b Non-invasive fetal ECG database: The non-invasive fetal ECG database consists of a series of 55 multichannel ECG recordings. The ECG signals in this dataset were taken from a subject between 21 and 40 weeks of pregnancy. Two of these simultaneous signals were obtained from the mother's thoracic region while other three or four were obtained from the mother's abdominal region with sampling frequency of $1 \mathrm{kHz}$ (Goldberger et al 2000).

Table 1 shows the Se, PPV, accuracy, standard deviation of accuracy $\left(\sigma_{\text {accuracy }}\right)$ and range of accuracy of Proposed method (EKS+ANFIS, EKF+ANFIS), EKS (par-EKS) (Niknazar et al 2013), EKF (Sameni 2008), non-parametric method (Niknazar et al 2012), sequential processing method (modified template subtraction) (Arias-Ortega \& Gaitán-González 2010), Singular Value Decomposition (SVD) (method 3) (Kanjilal et al 1997). It also shows that accuracy of EKS+ANFIS is better than other methods and $\sigma_{\text {accuracy }}$ of EKS+ANFIS is less than other methods.

Table 1. Comparison of the average se, average PPV and average accuracy for all record of non-invasive fetal ECG database with length $1 \mathrm{~min}$.

\begin{tabular}{lccccc}
\hline Method & $\begin{array}{c}\text { Se } \\
(\%)\end{array}$ & $\begin{array}{c}\text { PPV } \\
(\%)\end{array}$ & $\begin{array}{c}\text { Accuracy } \\
(\%)\end{array}$ & $\begin{array}{c}\sigma_{\text {accuracy }} \\
\text { (accuracy) }\end{array}$ & $\begin{array}{c}\text { Range } \\
\text { (accuracy) }\end{array}$ \\
\hline EKS+ANFIS & 93.81 & 95.97 & 90.20 & 3.05 & $83.8-97.6$ \\
EKF+ANFIS & 92.75 & 94.85 & 88.21 & 3.05 & $81.1-94.2$ \\
EKS (Par-EKS) (Niknazar et al 2013) & 92.56 & 93.57 & 86.95 & 3.14 & $79.1-92.5$ \\
Non-parametric method (Niknazar et al 2012) & 93.24 & 92.06 & 86.25 & 3.18 & $79.1-92.3$ \\
EKF (Sameni 2008) & 91.49 & 93.29 & 85.83 & 4.27 & $78-97.6$ \\
Sequential processing method (modified & 92.01 & 90.89 & 84.25 & 3.55 & $71.3-91.9$ \\
template subtraction) & & & & & \\
(Arias-Ortega \& Gaitán-González 2010) & & & & & \\
Singular value decomposition & 90.19 & 89.21 & 81.33 & 3.28 & $70.6-88.3$ \\
(SVD) (method 3) (Kanjilal et al 1997) & & & & & \\
\hline
\end{tabular}




\section{Conclusion}

In this paper, ANFIS is used with synthetic dynamic ECG model within a KF framework to extract fetal ECG. The proposed model uses a single channel to separate fetal ECG from abdominal ECG which is dominated by maternal component of ECG. As validated on synthetic data and illustrated on actual data, the merit behind the proposed algorithm relies on its performance in a large class of data. The performance of the proposed method on extraction of fetal ECG from single channel abdominal ECG has been tested for various noise levels. According to the obtained results, as long as R peaks are correctly detected, the proposed model claims its accuracy.

Finally the proposed technique (EKS+ANFIS) is focused to be more accurate compared to EKF, EKS, non-parametric method, sequential processing method (modified template subtraction) and SVD. This technique (EKS+ANFIS) is of high interest, since a single channel does not only require less electronic components but also treated as a more convenient and portable device for a long term monitoring system or at home.

In our proposed model we have used peak detection method to detect $\mathrm{R}$ peak, but for noisy environment or when abdominal ECG is a combination of more than two ECG, peak detection method is not suitable to detect $\mathrm{R}$ peak. So better techniques can be used to detect $\mathrm{R}$ peak. Here we have focused only to extract single fetal ECG. This idea can also be extended to extract multi fetal ECG studies.

\section{References}

Abboud S, Alaluf A, Einav S and Sadeh D 1992 Real-time abdominal fetal ECG recording using a hardware correlator. Comput. Biol. Med. 22: 325-335. doi:10.1016/0010-4825(92)90021-E

Arias-Ortega R and Gaitán-González M J 2010 Single channel abdominal ECG algorithm for real-time maternal and fetal heart rate monitoring. Revista Mexicana de Ingeniería Biomédica 32: 111-118

Assaleh Khaled 2007 Extraction of fetal electrocardiogram using adaptive neuro-fuzzy inference systems. IEEE Trans. Bio-med. Eng. 54: 59-68. doi:10.1109/TBME.2006.883728

Assaleh Khaled and Hasan Al-Nashash 2005 A novel technique for the extraction of fetal ECG using polynomial networks. IEEE Trans. Bio-med. Eng. 52: 1148-1152. doi:10.1109/TBME.2005.844046

Callaerts D, De Moor B, Vandewalle J, Sansen W, Vantrappen G and Janssens J 1990 Comparison of SVD methods to extract the foetal electrocardiogram from cutaneous electrode signals. Med. Biol. Eng. Comput. 28: 217-224. doi:10.1007/BF02442670

Camps G, Martinez M and Soria E 2001 Fetal ECG extraction using an FIR neural network. Comput. Cardiol. 2001. Vol 28 (Cat. No.01CH37287). doi:10.1109/CIC.2001.977639

Camps-Valls Gustavo, Marcelino Martínez-Sober, Emilio Soria-Olivas, Rafael Magdalena-Benedito, Javier Calpe-Maravilla and Juan Guerrero-Martínez 2004 Foetal ECG recovery using dynamic neural networks. Artif. Intell. Med. 31: 197-209. doi:10.1016/j.artmed.2004.03.005

Deans A C and Steer P J 1994 The use of the fetal electrocardiogram in labour. Br. J. Obstetrics Gynaecol. 101: 9-17

Ferrara E R and Widrow B 1982 Fetal electrocardiogram enhancement by time-sequenced adaptive filtering. IEEE Trans. Bio-med. Eng. 29: 458-460. doi:10.1109/TBME.1982.324973

Goldberger A L, Amaral L A, Glass L, Hausdorff J M, Ivanov P C, Mark R G, Mietus J E, Moody G B, Peng C K and Stanley H E 2000 PhysioBank, PhysioToolkit, and PhysioNet: Components of a new research resource for complex physiologic signals. Circulation 101: E215-E220. doi:10.1161/01.CIR.101.23.e215

Hasan M A, Ibrahimy M I and Reaz M B I 2007 Techniques of FECG signal analysis: Detection and processing for fetal monitoring. WIT Trans. Biomed. Health 12: 295-305. doi:10.2495/BIO070281 
Kakar Manish, Håkan Nyström, Lasse Rye Aarup, Trine Jakobi Nøttrup and Dag Rune Olsen 2005 Respiratory motion prediction by using the adaptive neuro fuzzy inference system (ANFIS). Phys. Med. Biol. 50: 4721-4728. doi:10.1088/0031-9155/50/19/020

Kanjilal P P, Palit S and Saha G 1997 Fetal ECG extraction from single-channel maternal ECG using singular value decomposition. IEEE Trans. Bio-med. Eng. 44: 51-59. doi:10.1109/10.553712

Khamene A and Negahdaripour S 2000 A new method for the extraction of fetal ECG from the composite abdominal signal. IEEE Trans. Bio-med. Eng. 47: 507-516. doi:10.1109/10.828150

De Lathauwer L, De Moor B and Vandewalle J 2000 Fetal electrocardiogram extraction by blind source subspace separation. IEEE Trans. Bio-med. Eng. 47: 567-572. doi:10.1109/10.841326

Manikandan Sabarimalai M and Soman K P 2012 A novel method for detecting R-peaks in electrocardiogram (ECG) signal. Biomed. Signal Process. Control 7: 118-128. doi:10.1016/j.bspc.2011.03.004

McSharry Patrick E, Gari D Clifford, Lionel Tarassenko and Leonard A Smith 2003 A dynamical model for generating synthetic electrocardiogram signals. IEEE Trans. Bio-med. Eng. 50: 289-294. doi:10.1109/TBME.2003.808805

Mochimaru F 2002 Detecting the fetal electrocardiogram by wavelet theory-based methods. Prog. Biomed. Res. 7: 185-193

Moor B De, De Gersem P, De Schutter B, Favoreel W and Bart De Moor 1997 DAISY: A database for identification of systems. Journal A 38: 4-5

Nallathambi Gabriel and Student Member 2014 Integrate and fire pulse train automaton for QRS detection. IEEE Trans. Biomed. Eng. 61: 317-326

Niknazar Mohammad, Bertrand Rivet and Christian Jutten 2012 Fetal ecg extraction from a single sensor by a non-parametric modeling. In: 20th European Signal Processing Conference (EUSIPCO 2012), 949953

Niknazar Mohammad, Bertrand Rivet and Christian Jutten 2013 Fetal ECG extraction by extended state Kalman filtering based on single-channel recordings. IEEE Trans. Biomed. Eng. 60: 1345-52. doi:10.1109/TBME.2012.2234456

Sameni Reza 2008 Extraction of fetal cardiac signals from an array of maternal abdominal recordings. Ph.D. thesis, Sharif University of Technology-Institut National Polytechnique de Grenoble

Sameni Reza, Mohammad B Shamsollahi, Christian Jutten and Gari D Clifford 2007 A nonlinear Bayesian filtering framework for ECG denoising. IEEE Trans. Bio-med. Eng. 54: 2172-2185. doi:10.1109/TBME.2007.897817

Tal Y and Akselrod S 1989 Fetal heart rate detection by a special transform method. In: Proc. Comput. Cardiol. 275-278. doi:10.1109/CIC.1989.130542

Welch Greg and Gary Bishop 2006 An introduction to the Kalman filter 7:1-16. doi:10.1.1.117.6808

Zarzoso V and Nandi A K 2001 Noninvasive fetal electrocardiogram extraction: Blind separation versus adaptive noise cancellation. IEEE Trans. Bio-med. Eng. 48: 12-18. doi:10.1109/10.900244

Zarzoso V, Nandi A K and Bacharakis E 1997 Maternal and foetal ECG separation using blind source separation methods. IMA J. Math. Appl. Med. Biol. 14: 207-225. doi:10.1093/imammb/14.3.207

Zeng Yanjun, Shijin Liu and Jianhua Zhang 2008 Extraction of fetal ECG signal via adaptive noise cancellation approach. In: 2008 2nd International Conference on Bioinformatics and Biomedical Engineering. doi:10.1109/ICBBE.2008.899 